\title{
Competitive Programming 4: The New Lower Bound of Programming Contests in the 2020s
}

\author{
Steven HALIM \\ School of Computing, National University of Singapore \\ Computing 1, 13 Computing Drive, Singapore 117417 \\ e-mail:dcssh@nus.edu.sg
}

\begin{abstract}
Seven years have passed since me and my brother Felix Halim released the 3rd edition of our Competitive Programming book (CP3) on 24 May 2013 that had influenced the competitive programming field in the past decade: $2010 \mathrm{~s}$. We have just released the 4th edition of our book (CP4) on 19 July 2020 - the original IOI 2020 arrival day where free preview copies should have been given to all invited delegations. In this short report, we address two groups of readers: those who have read/heard about $\mathrm{CP} 3$ and those who are new with this book.
\end{abstract}

Keywords: competitive programming, book, IOI, ICPC.

\section{The Impact of the Earlier Editions of Competitive Programming Book}

We first released Competitive Programming 1 (CP1) before IOI 2010 in Waterloo, Canada, and updated it one year later with the $2^{\text {nd }}$ edition (CP2) after IOI 2011 in Pattaya, Thailand. However, the most impactful and long-lasting edition so far was the $3^{\text {rd }}$ edition (CP3), released in 2013 before IOI 2013 in Brisbane, Australia (Halim and Halim, 2013). Before we wrote CP1, there is only one other existing English book in competitive programming: Programming Challenges (Skiena and Revilla, 2003).

We write Competitive Programming book with the main objective of improving the lower bound of the typical long tail of the distribution of worldwide (pre-)competitive programmers, i.e., secondary/high school students or freshmen in University who have just started basic programming and want to improve their data structures, algorithms, (competitive) programming techniques, and problem-solving skills.

In these past seven years (2013-2020), we have received numerous thank you emails (see selected testimonials at https://cpbook.net/) and also the annual requests for autographs and/or wefie whenever we met young CP3 readers in the recent IOIs or ICPC Regionals/World Finals. Many of these young readers found CP3 as a "catalyst" for 
their personal competitive programming growth. By reading $\mathrm{CP} 3$, these young readers can quickly learn about the knowledge needed to compete decently in the IOIs, e.g., the IOI syllabus (Forišek, 2019) and in the ICPC regionals. While not the original intention, many readers have also expressed gratitude that studying the material in CP3 helped them to secure lucrative jobs at top IT companies. The generally positive feedback from the readers motivated us to continue studying the recent trends in this fast-evolving competitive programming world, including to read the Guide to Competitive Programming book (Antii Laksonen, 2017 and 2020), and to continue this book writing project with a third author: Suhendry Effendy. Now in the year 2020, we are ready to release our latest $/ 4^{\text {th }}$ edition (CP4).

In the next section, we highlight the main differences between this impactful CP3 with the new CP4 for the new decade: 2020s.

\section{Comparison with CP3 (2013)}

This section is for the benefit of the reader who has read CP3 sometime in the past seven years and possibly a current active IOI/ICPC coach, a high school teacher in informatics, or a University lecturer that can influence future young $\mathrm{CP} 4$ readers.

The major change is the split of CP4 into two volumes: Book 1 is mostly for IOI (most content of the IOI syllabus (Forišek, 2019) are discussed here) + basic ICPC level and Book 2 is mostly for medium ICPC level. Secondary or high school students can start from CP4 Book 1 first and only move to Book 2 when they enter University.

Other major update is the addition of Kattis online judge (https : //open. kattis . com). Kattis has growing problem bank that includes IOI-related tasks, e.g., Croatian Open Competition in Informatics (COCI) tasks 2006-2017, including some newer problem types: interactive and constructive problems.

\begin{tabular}{|c|c|c|}
\hline Features & $\mathrm{CP} 3$ & CP4 \\
\hline Number of Books & 1 & Split into two books \\
\hline Number of Chapters & 9 & $1-4+5-9=9$ \\
\hline Number of Pages & 447 & $329+352=681(>1.5 x)$ \\
\hline Authors & Steven, Felix & Steven, Felix, Suhendry $(+1)$ \\
\hline $\begin{array}{l}\text { Authors combined } \\
\text { experiences }\end{array}$ & $\begin{array}{l}\text { ex IOI/ICPC Regionals+ } \\
\text { World Finals Contestants, } \\
\text { active coaches and problem } \\
\text { authors of recent programm- } \\
\text { ing contests }\end{array}$ & $\begin{array}{l}\text { + ICPC Asia Singapore Regional Contest Director } \\
(2015+2018) \text {, ten ICPC Asia Regional wins (as } \\
\text { coach), many more IOI gold+ silver+bronze } \\
\text { medals for team Singapore, IOI Deputy Director+ } \\
\text { International Committee member }(2020+2021)\end{array}$ \\
\hline Programming Languages & $\mathrm{C}++(11)$, Java (7) & $\begin{array}{l}\mathrm{C}++(17), \text { Java }(11), \text { and } \\
+2 \text { more: Python }(3), \text { OCaml }\end{array}$ \\
\hline Programming Exercises & UVa: 1675 & UVa+Kattis: 3458 (> 2x) \\
\hline Figures generated using & $\begin{array}{l}\text { PowerPoint }+ \text { older visuali- } \\
\text { zation tool }\end{array}$ & $\begin{array}{l}\text { Mostly VisuAlgo } \\
\text { (Halim, 2015) screenshots }\end{array}$ \\
\hline
\end{tabular}


Albeit not included in the IOI yet (but available in the ICPC), we have integrated discussion on when it is appropriate to use Python (3) programming language to solve competitive programming problems whenever it is allowed. Sample implementation code discussed in CP4 are available at https://github.com/stevenhalim/ cpbook-code

We also rewrite most sections and update many figures with the latest screenshots of our Visualization tool: VisuAlgo: https : //visualgo net (Halim, 2015), thus CP4 readers can further deepen their understanding of the data structure/algorithm being discussed by trying their input data at VisuAlgo together when reading CP4.

The major additions are the topics that were previously not written yet in CP3 but are now written in CP4 (many are outside the IOI syllabus (Forišek, 2019) and more appropriate for ICPC): Modular Multiplicative Inverse, String Hashing, Square Root Decomposition, Heavy-Light Decomposition, Tree Isomorphism, De Bruijn Sequence, Fast Fourier Transform, Chinese Remainder Theorem, Lucas' Theorem, Combinatorial Game Theory, Egg Dropping Puzzle, Dynamic Programming Optimization, Push-Relabel algorithm, Kuhn-Munkres algorithm, Edmonds' Matching algorithm, Constructive Problem, Interactive Problem, Linear Programming, Gradient Descent.

\section{For New CP4 Readers in 2020s}

The 2020s decade has just started (albeit with a global COVID-19 pandemic). We believe that CP4 Book 1 provides the necessary (but not necessarily sufficient) conditions needed to prepare the many young readers for their National Olympiad in Informatics (NOI) preparation leading to the IOI. Similarly, we also believe that CP4 Book $1+2$ provides the same necessary (but not necessarily sufficient) conditions needed to prepare many new University students for their ICPC Regionals leading to the ICPC World Finals.

The details on how to get a copy of this book via its various distribution channels can be found at the book's companion website: https://cpbook. net.

\section{References}

Forišek, M. (2019), http://people.ksp.sk/ 〜misof/ioi-syllabus/

Halim, S., Halim, F. (2013). Competitive Programming 3: The New Lower Bound of Programming Contests. Lulu.

Halim, S. (2015). VisuAlgo - Visualising Data Structures and Algorithms Through Animation. Olympiads in Informatics, 9, 243-245.

Laaksonen, A. (2017). A new book on competitive programming. Olympiads in Informatics, 11, 167-170.

Laaksonen, A. (2020). Guide to Competitive Programming: Learning and Improving Algorithms Through Contests. Second Edition. Springer.

Skiena, S.S., Revilla, M.A. (2003). Programming Challenges: The Programming Contest Training Manual. Springer. 




S. Halim is a senior lecturer in the School of Computing, National University of Singapore (SoC, NUS). He teaches several programming courses in NUS, ranging from basic programming methodology, intermediate to hard data structures and algorithms, web programming, and Competitive Programming. He is the coach of both the NUS ICPC teams and the Singapore IOI team. So far (2009-2019), he and other trainers at NUS have successfully groomed various ICPC teams that won ten different ICPC Regionals, advanced to ICPC World Finals eleven times with the current best result of Joint-14th in ICPC World Finals Phuket 2016, as well as seven gold, nineteen silver, and fifteen bronze IOI medalists. He is also the Regional Contest Director of ICPC Asia Singapore 2015+2018 and is the Deputy Director+International Committee member for the IOI 2020+2021 in Singapore. 